\title{
THE HIGH-VOLTAGE SYSTEM OF CALET APPARATUS
}

\author{
Francesco Petroni ${ }^{(1)}$, Lorenzo Fontani ${ }^{(1)}$, Luca Onida ${ }^{(1)}$, Pierpaolo Pergola ${ }^{(1)}$, \\ Guido Castellini (2), Andrea Viciani (1) $^{(1)}$ \\ (1) SITAEL, Via Livornese 1019, Pisa, Italy, Email: francesco.petroni@sitael.com \\ (2) IFAC CNR, Via Madonna del Piano 10, Firenze, Italy,Email: g.castellini@ifac.cnr.it
}

\begin{abstract}
CALET (CALorimetric Electron Telescope) is installed on the International Space Station since August 2015, is a mission devoted to measure the intensity of cosmic ray electrons and protons accelerated to near the speed of light. The apparatus is also designed to observe high energy gamma rays, nearby source of high energy radiation and it may even detect signatures of the elusive dark matter.

One of the core components of the whole apparatus is the high voltage power supply system. It is based on hundred of independent DC/DC converters providing precise voltage regulation to two sections of CALET detectors.

This paper presents the experience gained in the implementation of the CALET HV system: the system design requirements and the technical solutions adopted, the two system sections block schemes and the main characteristics of the DC/DC converters that guarantee the CALET HV system technical performance.
\end{abstract}

\section{The CALET experiment}

CALET is a sophisticated scientific apparatus for measurements of high-energy $(\mathrm{GeV}-\mathrm{TeV})$ fluxes of electrons and photons, and also protons and atomic nuclei, originating from outside the solar system. It has been developed within an international Japanese led cooperation involving nearly 50 researchers from Japan, Italy and the USA [1].

CALET aims at addressing several high-energy astrophysics questions: the origin of cosmic rays; how these accelerate and travel across our galaxy; evidence of the existence of dark matter; the origin of nearby cosmic-ray; etc. Furthermore, CALET expects also to provide an important insight of rare interactions between matter and dark matter due to its long permanence in space [2].

CALET will complete the observations of CREAM, PAMELA and AMS-02 on a possible deviation from a pure power-law of proton and He spectra from the few hundred $\mathrm{GeV}$ to multi-TeV region [2].

The module has been launched in orbit aboard the HTV5 vehicle and installed on the International Space Station (ISS) in 2014. CALET is currently attached to the Japanese Experiment Module (JEM), Kibo [3] . The main CALET telescope consists of an array of scintillation detectors to determine the electric charge of the incoming cosmic ray particles, an imaging calorimeter of scintillating fibers to determine particle trajectory, and a tungstate calorimeter to measure particle and gamma ray energies up to $20 \mathrm{TeV}$ [4], see Fig. 1.

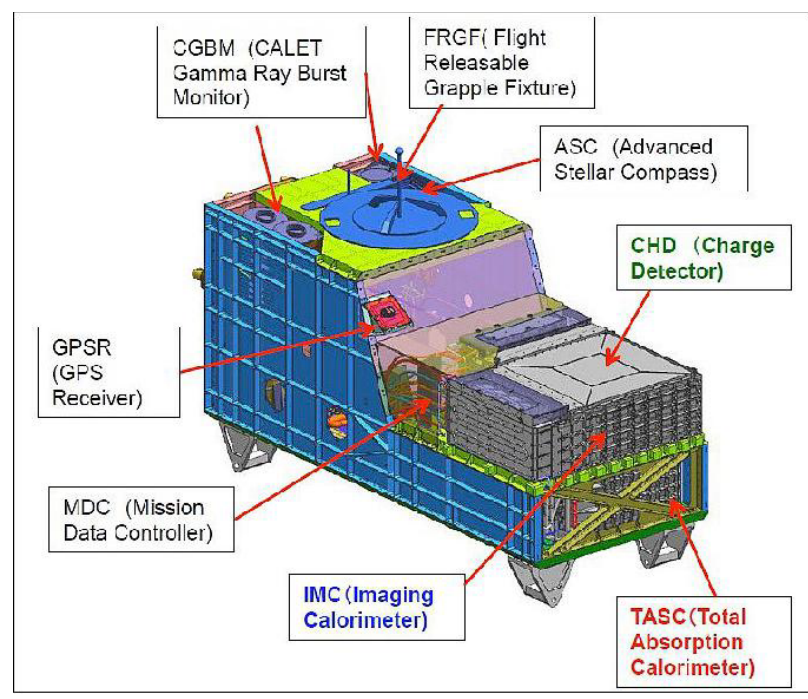

Figure 1. Overview of the main CALET telescope

The electromagnetic calorimeter has an approximately cubic size, $50 \mathrm{~cm}$ side length, and it is horizontally segmented in a set of layers alternating high-density and scintillating materials [5].

The working principle of the calorimeter is that a single high-energy charged particle or photon, traversing the high-density layers, produces a shower of secondary charged particles. These particles interact in the scintillating layers (scintillating fibres, lead tungstate PWO) generating scintillation light that is collected by different types of detectors:

- photomultiplier tubes (PMT);

- photodiodes (PD);

- avalanche photodiodes (APD).

The charge detector is composed of several layers of plastic scintillator, to be crossed by the particles before getting into the calorimeter, that provide information on intensity and on distribution of the scintillation signal. 
These data are thus collected to determine species and associated energy of the primary incident particle [5].

\section{The CALET HV system design criteria}

The CALET high-voltage (HV) system (surnamed brick) fulfils the task of generating the high voltages (from $500 \mathrm{~V}$ to $1000 \mathrm{~V}$ ) required for the operation of the PMT and APD photo-detectors.

The HV system has been designed and implemented by SITAEL in coordination with CNR-IFAC utilizing COTS components.

The HV system has been conceived, manufactured and tested to cope with the stringent requirements deriving from long time operations in space environment. Among these requirements, it is worth to mention:

- The brick structure was required to be stiff enough to support the mechanical stresses encountered during the launch phase;

- The materials used for each HV module was required to be compatible with the foreseen temperature and pressure conditions during orbital flight. For the qualification of the system temperatures between $-20{ }^{\circ} \mathrm{C}$ and +65 ${ }^{\circ} \mathrm{C}$ and pressures below $10^{-5}$ mbar have been used;

- The overall system designed was required to be compatible with the high level of ionizing radiation present in low-Earth orbit. Qualification up to at least $10 \mathrm{krad}$ of total absorbed ionizing dose, and immunity for electrical events caused by single ionizing particle (Single Event Effect, SEE) for the whole duration of the mission ( $>5$ years) were the two main drivers;

- The electromagnetic interference and compatibility (EMI/EMC) was required to cope with the stringent requirements of payloads installed on the ISS space station.

A first specimen of the HV system, the "HV Box 001" was delivered to JAXA in 2012, with the purpose of performing characterization and qualification. The "HV Box 002", to be integrated with the CALET apparatus and launched for flight operation, was completed and delivered to JAXA on April 2013.

\section{The CALET HV system configuration}

The CALET HV system is composed of an aluminium support structure $290 \times 240 \times 240 \mathrm{~mm}^{3}$, housing:

- 80 modules in the "PMT section" (derived from the design of AMS02 HV System) delivering a corresponding number of negative high-voltage lines (up to $-1000 \mathrm{~V}$ ) for the PMT photodetectors of CALET system;

- 22 modules in the "APD section", delivering a corresponding number of negative lines, which voltages up to $-500 \mathrm{~V}$, for the APD photodetectors;

- the related electronic boards (PHCB and AHCB) for control and interface with the Mission Data Controller (MDC) unit managing the operation of the CALET apparatus.

The HV brick features a total of 102 redundant independent $\mathrm{HV}$ lines. The system weights in total about $14 \mathrm{~kg}$ and its power consumption is approximately $22 \mathrm{~W}$ in the nominal operating configuration, see Fig. 2
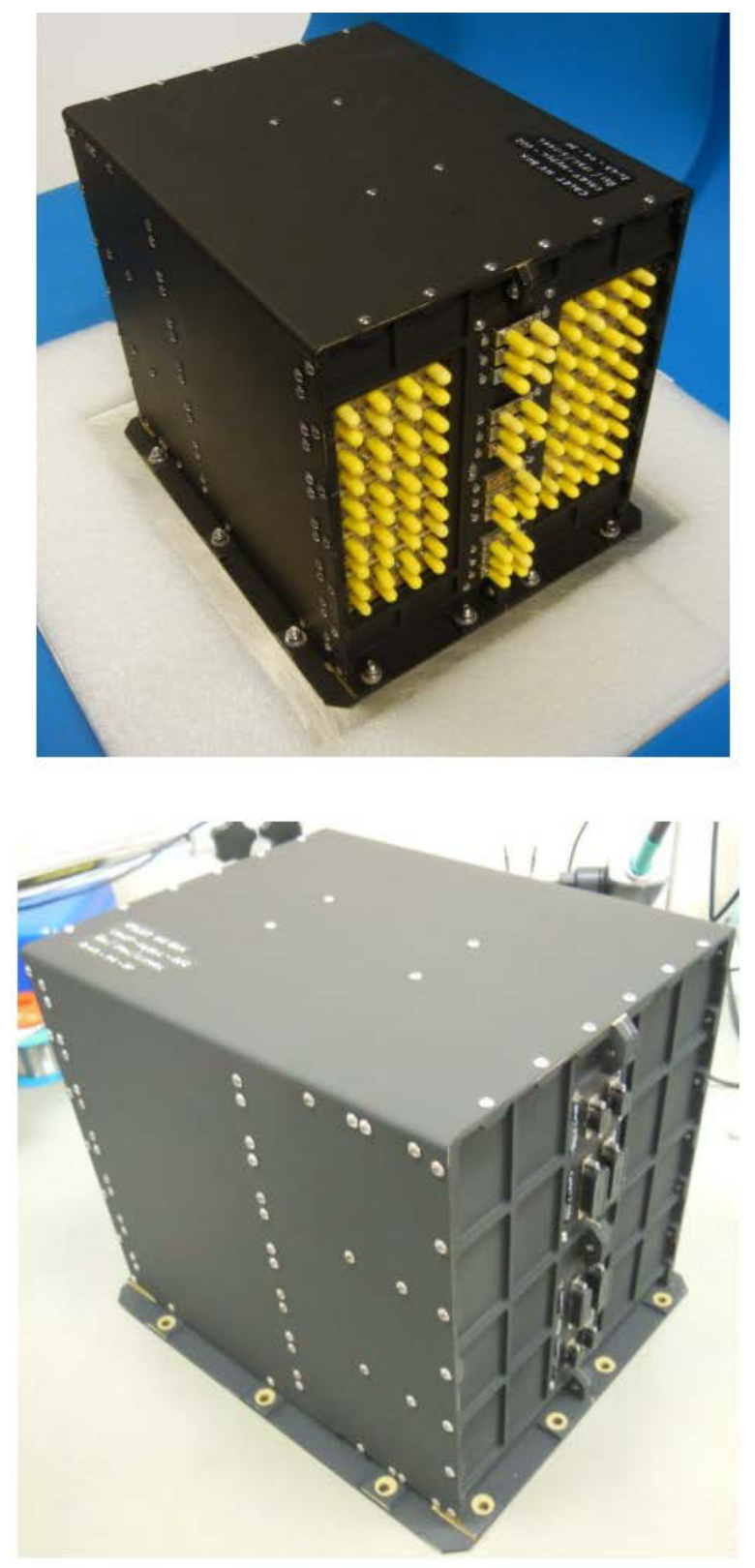

Figure 2. The CALET HV system front (up) and back (bottom)

On the front side of the HV unit, 102 miniaturized HV 
coaxial connectors are installed for the connection of each line. On the rear side a total of 4 power connectors are installed on the mechanical structure for input power lines and digital signals.

The PMT and APD sections are physically divided into two electrically identical halves (Fig. 3). Each half is composed of a hot and a cold (identical) part for redundancy: each DC/DC and Linear Regulator (LR) is doubled, while each control board is formed by two parts (hot and cold part).

The doubled parts are identified as hot (normally powered-on) and cold (normally powered-off and used for substitution in the case of unrecoverable damage). When the hot HV output of a given channel (LR or $\mathrm{DC} / \mathrm{DC}$ ) is on, the corresponding cold HV output has been turned off, or vice versa. The hot and cold part of each control board can be powered independently (with commands addressing each part independently).

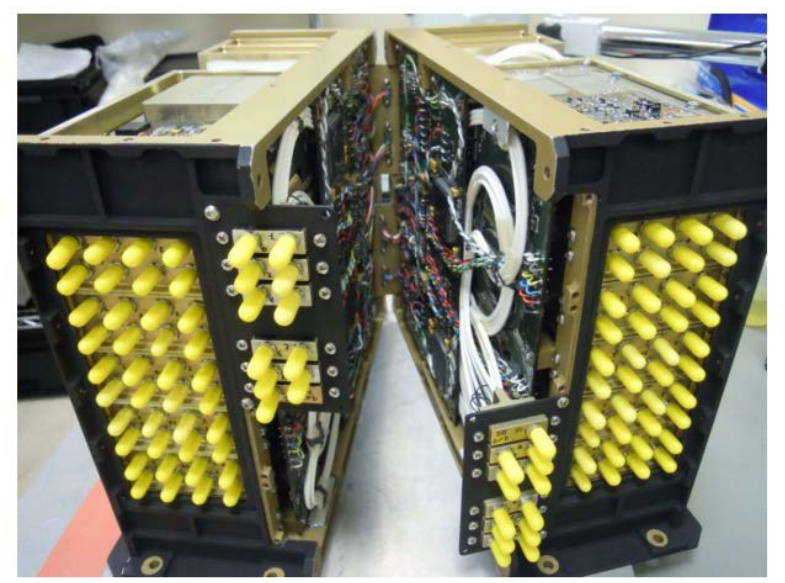

Figure 3. The CALET HV system (PMT and APD sections) open in its two halves

The PMT HV section of each brick contains two (hot/cold) DC/DCs and 5 (hot/cold) groups (boxes) each composed of $8 \mathrm{LRs}$, and correspondingly a total of 40 HV output channels (one HV connector per channel). Each (hot/cold) LR generates a typical HV (varying with channel).

The APD HV section of each brick is formed by a total of 11 (hot/cold) DC/DC converters, driving 11 FEC channels for a total of 88 APD's.

The converters are grouped into 4 mechanical boxes: two boxes contain 6 (hot/cold) converters each, while the other two boxes contain 5 (hot/cold) converters each. The boxes are equally divided between the two bricks, in such a way that each brick contains one box with 6 hot/cold converters and one box with 5 hot/cold converters.

The HV outputs of each hot/cold pair of LR's (for PMT section) or DC/DC's (for APD section) are ORed through a diode protection circuit, thus producing a single HV output channel.

The block schemes for half PMT and APD section is illustrated in Fig. 4. The HV system is designed to be interfaced with the Mission Data Controller (MDC) unit managing the sequence of operation of the whole CALET apparatus.

Up to three different power voltages are required to be provided by each section of the CALET HV system. The digital interface, for data and commands is implemented with LVDS bidirectional serial links, with a typical transmission rate of 1 Mbps. The HV lines have been designed and characterized for a linearity better than 3\% and a peak-to-peak noise (ripple) less than $150 \mathrm{mV}$.
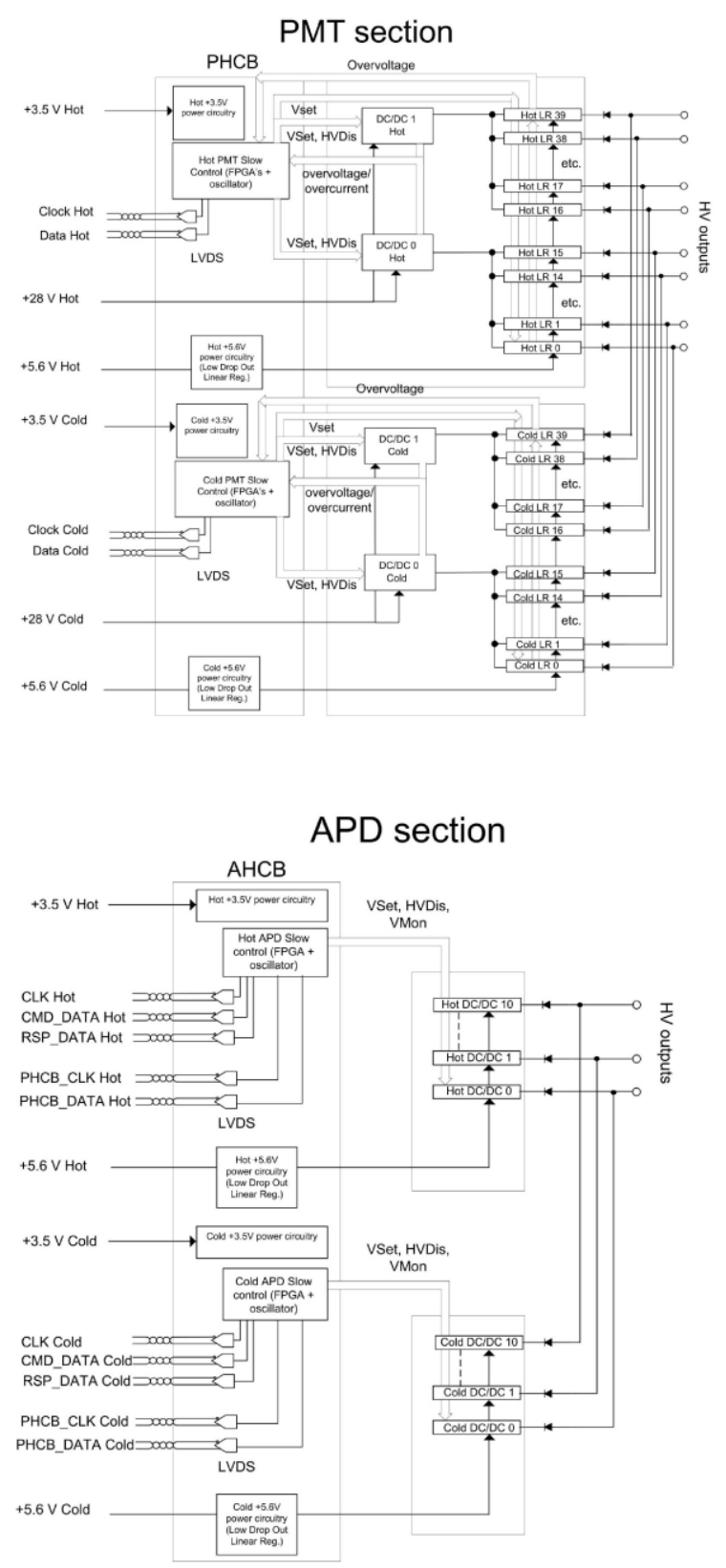

Figure 4. Block schemes for half PMT and APD section of the CALET HV system

The logic structure of the HV system has been 
implemented by means of FPGA programmable logic devices on the PHCB and AHCB (see fig.4) boards.

The FPGA family used in the PHCB is the Actel A54SX-A. This "antifuse" FPGA family guarantees correct and reliable operations in space under the foreseen radiation environment, without cumulative effects from absorbed Total Ionization Dose (TID) and a negligible rate of Single Event Effects (SEE), specifically SEU and SEL.

For the AHCB the considered FPGA family is the Actel ProASIC3E. The ProASIC3E family features the "flash programmable" technology, implementing the configuration memory of the FPGA logic structure on non-volatile flash EEPROM bits. This technology allowed for fast and zero-cost reprogramming on the same chip during the development phase. The FPGA features similar performances (and it is produced by the same vendor) to those of the A54SX-A family for what concerns radiation effects.

In general the logic architecture allows to:

- power on/off a single HV line or groups of HV lines;

- set the output voltage for each HV line, with $0.1 \%$ resolution over the whole dynamic range;

- real-time monitor the output voltages for the APD section;

- save and read the configuration of single lines;

- real-time detection of anomalous conditions, (overvoltage or overcurrent in the high-voltage modules, or SEE );

- automatically configure (at system power-on) the different $\mathrm{HV}$ lines, without needing to receive any external command, according to a scheme defined on the basis of the characteristics of the PMT or APD channels.

\section{The CALET HV DC/DC converters}

The power lines for PMT HV system are: $+28 \mathrm{~V} \mathrm{DC}$, $+5.6 \mathrm{~V} \mathrm{DC}$ and +3.5 V DC (nominal values). The power lines for APD HV system are: +5.6 V DC and +3.5 V DC (nominal values).

The DC/DC converter and linear regulators adopted for the PMT HV system are the same utilized in the past for AMS02 experiment.

The DC/DC converter adopted for each line of the APD HV system is a variation of the SITAEL S9097/8 family (more than 1000 flight module delivered mostly for the Chinese market in the last decade), modified (S9106) in such a way to have a reduced output voltage range lower than the $3000 \mathrm{~V}$ range as per specifications of these modules. Fig. 5 presents a string of 6 modules before assembly into the CALET brick.

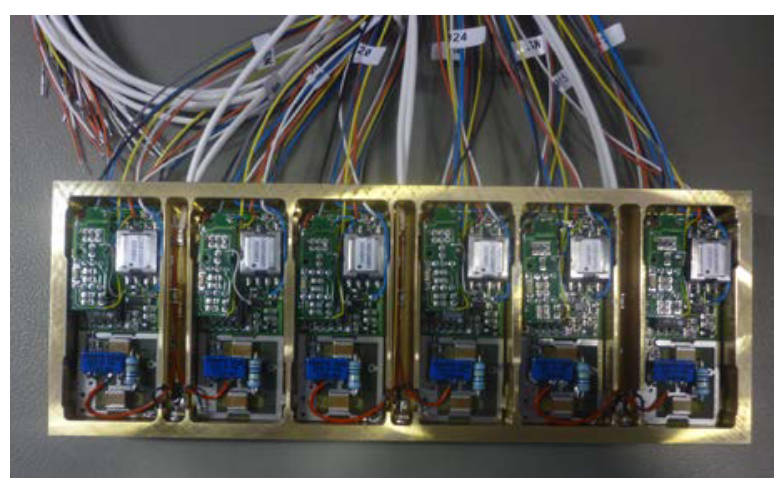

Figure 5. String of S9106 modules assembled in the CALET configuration

The S9106 modules ensure a minimum radiation tolerance of $30 \mathrm{Krad}$. The converter offers compact size, low power consumption, wide operating temperature range $\left(-40\right.$ to $\left.+70{ }^{\circ} \mathrm{C}\right)$, low ripple and high temperature stability. S9106 modules operate with a $+5 \mathrm{~V}$ DC input and deliver a remotely programmable output voltage ranging from 0 to $500 \mathrm{~V}$; the $\mathrm{DC} / \mathrm{DC}$ converter is provided with an output voltage monitor and an $\mathrm{HV}$ disable feature. Fig.6 presents the S9106 block diagram.

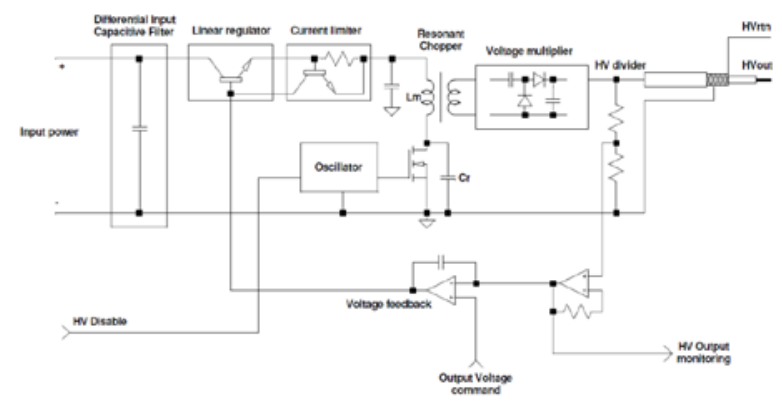

Figure 6. S9106 block diagram in the CALET configuration

More in detail the S9106 converters feature:

- capacitive input filter, which reduce the current ripple and noise injected into input bus;

- primary current limiter which intervenes in case of anomalous absorbed current;

- linear regulator which modulates the chopper voltage amplitude according to feedback signal;

- resonant chopper which is used to drive the step-up transformer with low switching losses

- voltage multiplier which raises the transformer voltage up to the desired output level;

- voltage monitor which senses the output voltage for both internal voltage feedback circuit and external output voltage monitor;

- voltage feedback which comparing the output voltage monitor and the set voltage, drives the linear regulator;

- voltage set which programs the output high 
voltage;

- HV Disable, which can disables the output voltage generation.

The switching converter works at fixed frequency, avoiding any unpredictable noise due to frequency beat which may increase noise level.

The resonant chopper has been implemented by adopting a ZVS technique (switching turn-on takes place at Zero Voltage) and ZCS technique (turn-off takes place at Zero Current) to drastically reduce switching losses, increment efficiency and lower conducted and radiated noise. The resonant condition will be maintained over the entire load range.

A 3-cell Cockroft-Walton voltage multiplier is used to lift the secondary voltage of the transformer up to the required output voltage. The usage of components with lower rated voltage than the output voltage yields mass and volume saving and high efficiency.

The use of a linear regulator to control the output voltage not only simplifies the circuit with respect to classic PWM solution but allows to employ a fixed frequency resonant chopper and to greatly ease feedback compensation network design since the converter behaves like a first order system.

S9106 configured for the CALET experiment is able to operate at any pressure, including the $0.1 \mathrm{mbar}$ to $10 \mathrm{mbar}$ range (minimum of Paschen curve), without any HV discharge or corona effect.

\section{Conclusions}

The HV power supply system for the Calorimetric Electron Telescope is one of the biggest ever realized for a space mission with more than 200 independently regulated $\mathrm{HV}$ channels designed and tested to behave in any vacuum condition. COTS components selected and utilized for PAMELA (in orbit since 10 years), AMS02 (in orbit since 5 years) and CALET (in orbit since August 2015) have shown outstanding characteristics and reliability as well as the compromise solutions adopted for the assembly. These successful criteria will be proposed as a baseline for SITAEL low cost line of products dedicated to micro and mini satellites for LEO missions.

\section{REFERENCES}

1. Torii, S., (2011), Overview of the CALET Mission to the ISS, 32nd International Cosmic Ray Conference, Beijing, China

2. Torii, S., et al. (2005), The CALET Instrument for Experiment on the ISS, 29th International Cosmic Ray Conference, Pune, India

3. Torii, S., (2015) The CALorimetric Electron
Telescope (CALET) on the ISS for High Energy Astroparticle Physics, ISPC ISS Research Investigations and Experiments, IKI RAS/Moscow, Russia

4. Marocchesi, P. S., (2015) CALET: a high energy astroparticle physics experiment on the ISS, World Scientific Publishing Co., arXiv:1512.08059 [astroph.IM]

5. Bigongiari, G., (2015) CALET perspectives for calorimetric measurements of high energy electrons based on beam test results, Proceedings of Science, 34th International Cosmic Ray Conference, The Hague, The Netherlands 